\title{
Measurements and Simulations of Ionization Chamber Signals in Mixed Radiation Fields for the LHC BLM System
}

\author{
B. Dehning, Ch. Fabjan, G. Ferioli, E.B. Holzer, M. Stockner. \\ CERN - Geneva - Switzerland
}

\begin{abstract}
The LHC beam loss monitoring (BLM) system must prevent the super conducting magnets from quenching and protect the machine components from damage. The main monitor type is an ionization chamber. About 4000 of them will be installed around the ring. The lost beam particles initiate hadronic showers through the magnets, which are measured by the monitors installed outside of the cryostat around each quadrupole magnet. They probe the far transverse tail of the hadronic shower. The specification for the BLM system includes a factor of two absolute precision on the prediction of the quench levels. To reach this accuracy a number of simulations are being combined to calibrate the monitor signals. To validate the monitor calibration the simulations are compared with test measurements. This paper will focus on the simulated prediction of the development of the hadronic shower tails and the signal response of ionization chambers to various particle types and energies. Test measurements have been performed at CERN and DESY and compared to Geant4 simulations.
\end{abstract}

Presented at IEEE $2006-29^{\text {th }}$ Oct $-4^{\text {th }}$ Nov 2006 -San Diego/CA - USA 


\title{
Measurements and Simulations of Ionization Chamber Signals in Mixed Radiation Fields for the LHC BLM System
}

\author{
Markus Stockner, Bernd Dehning, Christian Fabjan, Gianfranco Ferioli, and Eva Barbara Holzer
}

\begin{abstract}
The LHC beam loss monitoring (BLM) system must prevent the super conducting magnets from quenching and protect the machine components from damage. The main monitor type is an ionization chamber. About 4000 of them will be installed around the ring. The lost beam particles initiate hadronic showers through the magnets, which are measured by the monitors installed outside of the cryostat around each quadrupole magnet. They probe the far transverse tail of the hadronic shower. The specification for the BLM system includes a factor of two absolute precision on the prediction of the quench levels. To reach this accuracy a number of simulations are being combined to calibrate the monitor signals. To validate the monitor calibration the simulations are compared with test measurements. This paper will focus on the simulated prediction of the development of the hadronic shower tails and the signal response of ionization chambers to various particle types and energies. Test measurements have been performed at CERN and DESY and compared to Geant4 simulations.
\end{abstract}

Index Terms-LHC BLM, beam loss monitoring, Geant4.

\section{INTRODUCTION}

$\mathbf{T}$ HE LHC is a proton collider with injection energy of $450 \mathrm{GeV}$ and top energy of $7 \mathrm{TeV}$. Two counter rotating beams are crossing each other at the interaction points (IP). The stored energy is $360 \mathrm{MJ}$ maximum per beam (enough to melt $500 \mathrm{~kg}$ of copper) and $10 \mathrm{GJ}$ in the magnet system [1]. Lost beam protons can quench the magnets or even destroy machine components. There are several safety systems to protect the machines components from damage. One of them is the Beam Loss Monitoring (BLM) system that measures the lost beam protons outside of the magnet cryostats [2]. The main detector type is an ionization chamber. About 4000 will be installed. At special locations (e.g. collimator regions) additional secondary emission monitors (SEM) are used to increase the dynamic range from $10^{8}$ to $10^{13}$. The detectors probe the transverse tails of the hadronic showers through the cryostats which are induced by lost beam particles.

The start-up calibration of the BLM system is designed to be within a factor of five in accuracy and for the final calibration a factor of two is required. A number of simulations were performed to calibrate the system. The beam particles were tracked to find the most propable loss locations. At these loss locations hadronic showers through the machine components

Manuscript received November 17, 2006.

M. Stockner is with CERN and Vienna Univerity of Technology. All authors are with CERN, CH-1211 Geneva 23, Switzerland (e-mail: firstname.lastname@cern.ch).

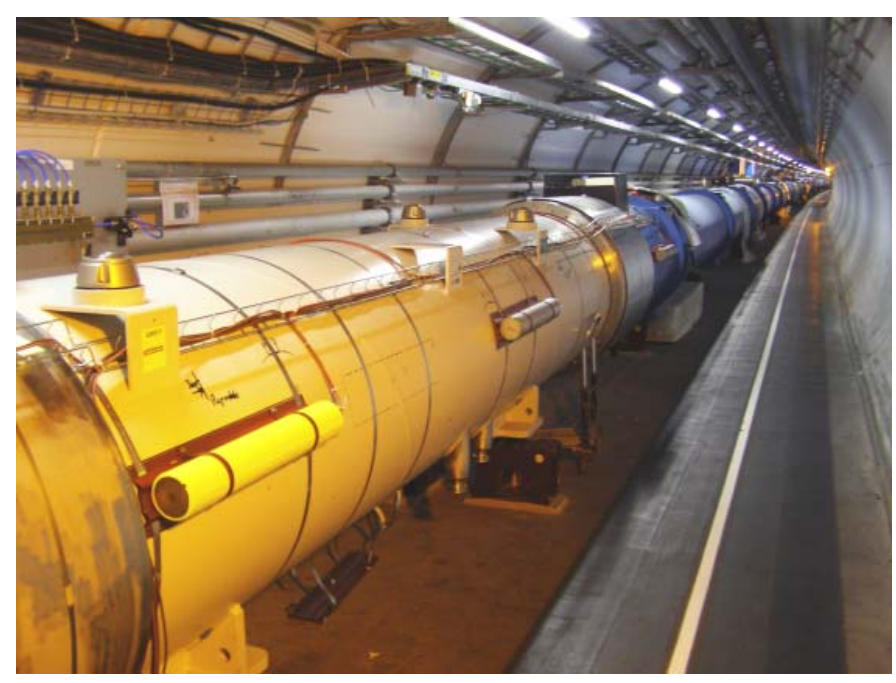

Fig. 1. LHC beam loss monitors (yellow insulation) mounted on a red support outside on a cryostat. They are horizontally aligned to the beam pipe.

are simulated to get the particle spectra at the detector locations. The spectra will be further used to simulate the detector signal. The quench levels of the superconducting magnets, according to loss duration and beam energy, are simulated separately. This paper will focus on the detector response simulation, which is part of the system calibration and on the uncertainty estimation of transverse hadronic shower tail simulations, which is part of the system calibration error. The simulations are verified by measurements performed at CERN and DESY.

\section{IONIZATION ChAmber Response Simulation}

Signal speed and robustness against aging were the main design criteria for the detectors.

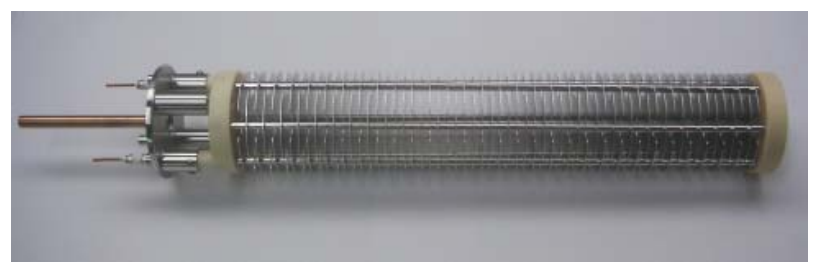

Fig. 2. Inside structure of the LHC BLM (ionization chamber).

Because of the high dynamic range an ionization chamber and a secondary emission monitor will be used. 
This paper will focus on the ionization chambers with parallel aluminum electrode plates separated by $0.5 \mathrm{~cm}$, as shown in Fig. 2. The detectors are about $50 \mathrm{~cm}$ long with a diameter of $9 \mathrm{~cm}$ and a sensitive volume of 1.5 liter. The collection time of the electrons and ions is of the order of $300 \mathrm{~ns}$ and $80 \mu$ s respectively. The chambers are filled with $\mathrm{N}_{2}$ at 100 mbar overpressure [2].

Depending on the loss location the detectors will be exposed to different radiation fields. The energy of the particles is spread over a large range from $\mathrm{keV}$ to $\mathrm{TeV}$ and their number is exponentially decreasing with energy.

Geant4 (version 8.0 patch-01) [3] simulations of the ionization chambers were performed to determine the signal response for different particle types at various kinetic energies in the range of $10 \mathrm{keV}$ to $10 \mathrm{TeV}$. Longitudinal (Fig. 3) and transverse (Fig. 4) impacting directions in respect to the detector axis were simulated. The longer path for a longitudinal direction increased the response approximately by a factor of two. Less wall material has to be passed for the transverse direction, that leads to a lower energy cut-off. The deposited energy in the sensitive volume was converted with the so called $\mathrm{W}$-value to the number of produced charges. The $\mathrm{W}$-value for $\mathrm{N}_{2}$ is $35 \mathrm{eV}$ per electron-ion pair [4].

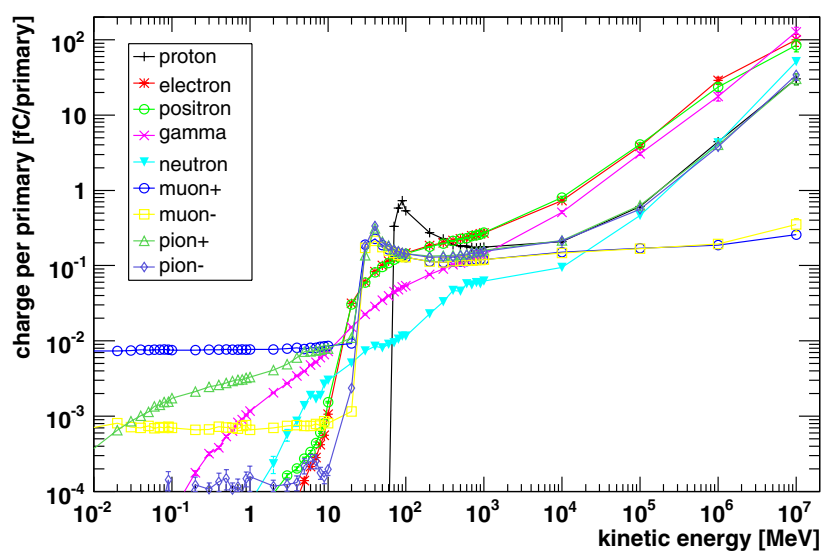

Fig. 3. Response of the ionization chamber for particles impacting longitudinally to the detector axis.

The following parameters in the simulation were varied to identify different contributions to the systematic error. The impacting angle causes a variation of the detector response; at high energy up to a factor of 100 for protons. The standard production range cut $(1 \mathrm{~mm})$ in Geant 4 was changed to $10 \mu \mathrm{m}$ (one fiftieth of the electrode thickness). This increased the detector response by $12 \%$. A further decrease to smaller values $(1 \mathrm{~nm})$ would increase the results additionally by $3 \%$, but increases the CPU time further.

The sensitive volume was determined by simulation of the electric field configuration. It is $4 \%$ bigger than the volume covered by the electrodes ( $2 \mathrm{~mm}$ larger diameter). NIST data were used to cross check the simulation [5]: The energy cut-off for protons, electrons and gamma rays was estimated. Protons of about $65 \mathrm{MeV}$ start to produce a signal, electrons at $9 \mathrm{MeV}$ and gammas at $150 \mathrm{keV}$ (parallel impact to detector axis).

The energy deposition for a positive muon was calculated with the Bethe-Bloch formula and compared to the simulation (agreement at $1 \mathrm{GeV}$ : $95 \%$ and at $35 \mathrm{MeV}: 75 \%$ ).

The strong rise in the detector response for high kinetic energies, in case of longitudinal impact (Fig. 3), was investigated. Depending on the relative impacting point of the particles to the inner structure more or less dense material has to be crossed. In denser material a larger number of interactions occur and the relative signal rises (e.g. stainless steel rods holding the electrodes).

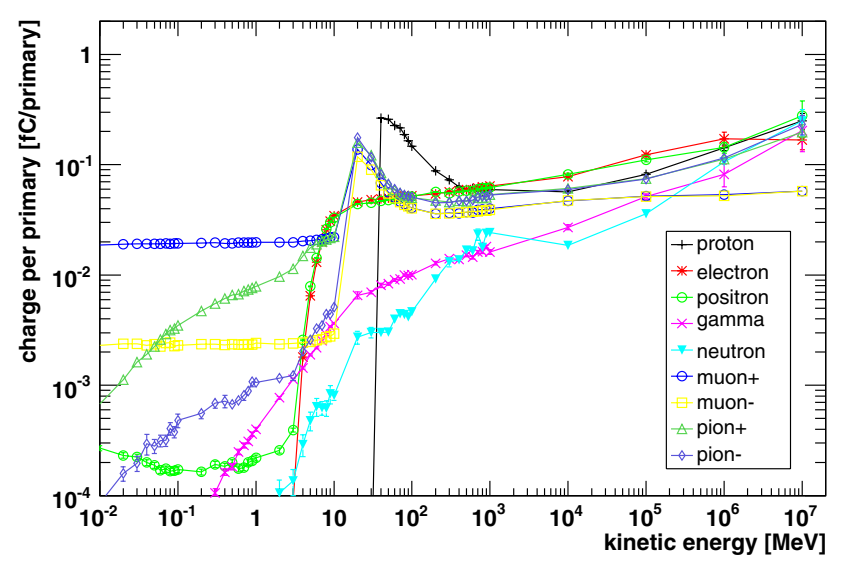

Fig. 4. Response of the ionization chamber for particles impacting transversely to the detector axis.

\section{VERIFICATION MEASUREMENTS}

Three measurements with different particle types and energies were performed to validate the Geant 4 simulations.

\section{A. Mixed Radiation Field Measurement}

A mixed radiation field experiment at the CERF target area (CERN-EU High Energy Reference Field Facility) was compared to the simulation. A copper target (length $50 \mathrm{~cm}$, diameter $7 \mathrm{~cm}$ ) was placed in a secondary beam of $120 \mathrm{GeV} / \mathrm{c}$ hadrons. The main beam particles were pions $(60.7 \%)$, protons $(34.8 \%)$ and kaons $(4.5 \%)$ with intensities up to $9.5 \cdot 10^{7}$ hadrons per 4.8 seconds. Five ionization chambers were positioned around the copper target so that they are exposed to different radiation fields (varying in particle composition and energy).

The CERF team had performed a similar experiment with PMI (air filled plastic ionization chamber) detectors and verified it by FLUKA simulations [6]. Their FLUKA spectra were used as input to simulate the detector response with Geant4. A comparison of the Geant 4 simulation to the BLM detector measurement shows a relative difference of about $12 \%$, except at detector position 1 (Table I). There, a relative difference of $21 \%$ can be seen. The detector specific energy cut-off and the shift of the particle spectrum to lower energies (below 
TABLE I

RESULT OF GEANT4 SIMULATIONS, BEAM MEASUREMENTS AND THEIR COMPARISON AT THE CERF TARGET AREA

$\left(10^{-12}\right.$ COULOMB PER $9.2 \cdot 10^{7}$ HADRONS $)$.

\begin{tabular}{|c|c|c|c|c|c|c|}
\hline & \multicolumn{2}{|c|}{ Geant4 sim. [pC] } & \multicolumn{2}{c|}{ measurement [pC] } & \multicolumn{2}{c|}{ ratio } \\
\hline pos. & SPS BLM & error & SPS BLM & error & sim/meas. & error \\
\hline \hline 1 & 91.13 & 0.35 & 115.33 & 11.66 & 0.79 & 0.08 \\
2 & 281.22 & 5.98 & - & - & - & - \\
3 & 1656.38 & 18.21 & 1577.75 & 162.59 & 1.05 & 0.11 \\
4 & 2386.62 & 21.53 & 2121.52 & 230.69 & 1.12 & 0.12 \\
5 & 3943.99 & 23.12 & 3531.98 & 370.42 & 1.12 & 0.12 \\
6 & 6495.5 & 17.54 & 7091.16 & 1096.82 & 0.92 & 0.14 \\
\hline
\end{tabular}

$1 \mathrm{GeV}$ ) leads to a low statistics in the number of particles that contribute to the detector signal. A comparison of the spectra between position 1 and position 6 is shown in Fig. 5. The error on the measurement includes the statistical error, a systematic error from uncertainties on the beam intensity measurement (10\%) and from misalignment investigations on the detector positions [6]. The error on the simulation includes only the statistical error of the signal simulation, it does not include the uncertainties in the spectrum.

All detectors showed a linear behavior at measurements over one order of magnitude in beam intensity (up to $9.5 \cdot 10^{7}$ hadrons onto the copper target). A first order polynomial fit showed for the detectors 3 to 6 a $\chi^{2} / n d f$ between $5 / 5$ and $10 / 5$, and for detector 1 a $\chi^{2} / n d f$ of $36 / 5$.
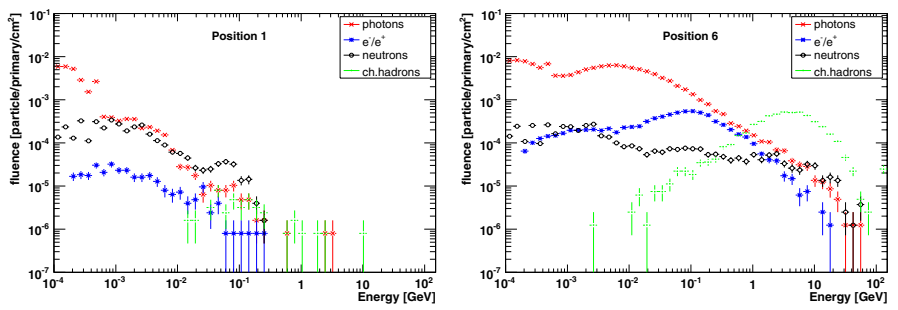

Fig. 5. FLUKA spectra for up- and down-stream position at the CERF target area [6].

\section{B. $400 \mathrm{GeV/c}$ Proton Measurement}

A second experiment with $400 \mathrm{GeV} / \mathrm{c}$ protons at an SPS extraction line (T2) was compared to the simulation. The beam size was estimated to $1 \mathrm{~cm}$ horizontally and $0.5 \mathrm{~cm}$ vertically $(4 \sigma)$. The intensity was $30.0 \cdot 10^{11} \pm 0.1 \cdot 10^{11}$ protons per 4.8 seconds. With Geant 4 a vertical scan of the beam position in the simulation was compared to the measurement. The unknown beam position (vertically) relative to the inner structure (parallel electrodes) led to a systematic uncertainty of $23 \%$. Measurement and simulation agree within errors (Table II).

\section{Gamma Ray Measurement}

A third comparison between simulation and measurement was done for gamma rays at the TIS-RP Calibration Laboratory
TABLE II

$400 \mathrm{GeV} / \mathrm{C}$ Proton Measurement Results (CHARGES PER PROTON PER CM).

\begin{tabular}{|c|c|c|c|c|c|}
\hline \multicolumn{2}{|c|}{ simulation $[\mathrm{q} /(\mathrm{p} \cdot \mathrm{cm})]$} & \multicolumn{2}{c|}{ measurement $[\mathrm{q} /(\mathrm{p} \cdot \mathrm{cm})]$} & \multicolumn{2}{c|}{ ratio } \\
\hline BLM & sys. error & BLM & error & sim./meas. & error \\
\hline \hline 124.84 & 25 & 110 & 0.06 & 1.13 & 0.23 \\
\hline
\end{tabular}

for Radiation Protection Instruments (CERN). The measurement was done with Cs137 sources at various activities and distances. The detector showed once more a linear behavior within two orders of magnitude in dose rate $(3 \mu \mathrm{Sv} / \mathrm{h}$ to $3 \mathrm{mSv} / \mathrm{h})$. The response simulation results for $600 \mathrm{keV}$ and $700 \mathrm{keV}$ gamma rays were interpolated and compared to the measurement results. The measurement and the simulation agree within $64 \%$ with an error of $7 \%$ (Table III).

TABLE III

GAMmA RAY MEASUREMENT RESUlts $\left(10^{-18}\right.$ COUlOMB PER PHOTON).

\begin{tabular}{|c|c|c|c|c|c|}
\hline \multicolumn{2}{|c|}{ simulation $[\mathrm{aC} / \gamma]$} & \multicolumn{2}{c|}{ measurement $[\mathrm{aC} / \gamma]$} & \multicolumn{2}{c|}{ ratio } \\
\hline BLM & error & BLM & error & sim./meas. & error \\
\hline \hline 0.27 & 0.02 & 0.42 & 0.01 & 0.64 & 0.05 \\
\hline
\end{tabular}

\section{Neutron Measurement}

Further verification and calibration measurements are planed for November 2006 at the Svedberg Laboratory, Uppsala University (Sweden) [7].

\section{HADronic Shower MEAsurements AT HERA}

The LHC BLM system start-up calibration is based on Geant3 and Geant4 simulations. Hadronic showers through LHC components and the detector response were simulated. An experiment at the HERA proton beam dump was set up to estimate the error on the far transverse hadronic shower tail simulations with Geant4. Six ionization chambers were installed on top of the dump, with a longitudinal spacing of about $1 \mathrm{~m}$. At this parasitic experiment the far transverse tails of hadronic showers can be measured. The impacting protons have energies of $40 \mathrm{GeV}$ (injection) and $920 \mathrm{GeV}$ (top energy). The intensity is in the range of $1.3 \cdot 10^{11}$ to $1.3 \cdot 10^{13}$ protons. The estimated error on the transverse hadronic shower tail simulations will be part of the BLM system calibration error. The simulation was split into two parts. First, the primary proton beam onto the dump was simulated and all particles arriving at the top of the dump were scored. In the second part, these secondary particles were launched for each detector position to get the detector signal. Two vertically separated impacting points on the dump were chosen to simulate the sweeping of the protons. The simulations were performed with Geant 4 8.0 (patch-01) and the QGSP physics list. The LHC BLM electronics was used to measure the detector signal. It consists of a CFC (current to frequency converter) and an FPGA (field 
programmable gate array) board, which provides the signal integrated over time windows, ranging form $40 \mu \mathrm{s}$ to $80 \mathrm{~s}$ [8]. The short particle pulse (one HERA turn corresponds to $21 \mu \mathrm{s}$ ) hitting the ionization chamber creates a high current (up to 1 A). A large filter $(\tau=\mathrm{R} \cdot \mathrm{C}=170 \mathrm{~ms})$ had to be installed in the input chain as the maximum input current to the electronic is $1 \mathrm{~mA}$.

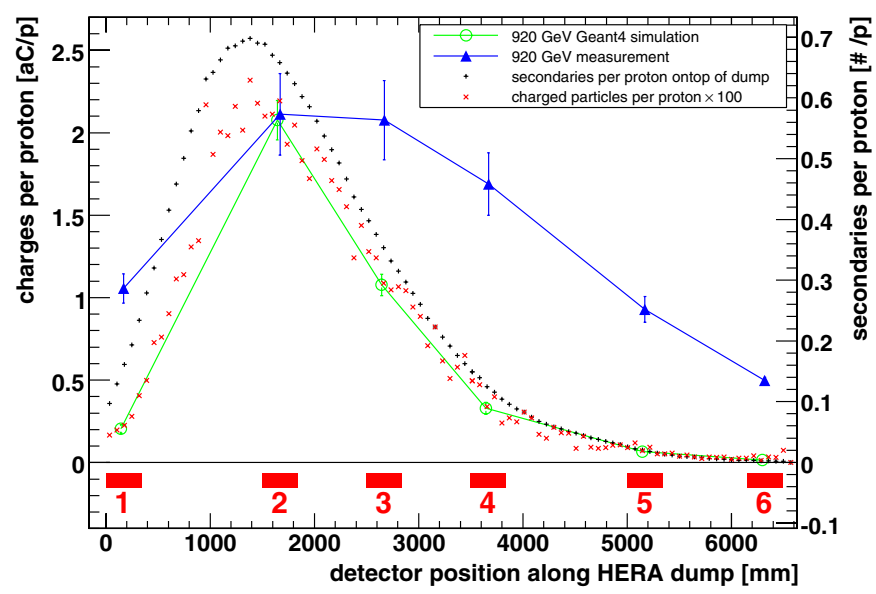

Fig. 6. Detector signal from simulation and measurement versus detector position on the HERA proton beam dump (preliminary data). Detector positions (in scale) at the bottom of the plot.

The simulation and measurement is in good agreement close to the shower maximum (detector position 2 in Fig. 6). The disagreement for the other detectors is not yet understood. It can be seen from Fig. 6 that uncharged particles dominate the spectra (less than $1 \%$ charged particles). Further investigations with different physics lists are planed, e.g. QGSP_BERT_HP (bertini models, high precision neutron transport) or QGSP_BIC_HP (binary cascade, high precision neutron transport), to identify possible systematic errors. Simplifications in the simulation were made: The beam size, the impacting angle $(\approx 1.5 \mathrm{mrad})$ and the tunnel geometry were not taken into account. These may also lead to greater uncertainties. The calibration of the readout electronic as function of input current led to a correction of maximal 20\%. A final electronics calibration has to be done. Recombination effects due to the short high intensity pulses will also be subject of further investigations.

\section{CONCLUSION}

The Geant4 detector response simulations are part of the LHC BLM calibration. The simulations were successfully performed and verified by different measurements. At the CERF target area the detector response in a mixed radiation field was compared to Geant 4 simulations. The agreement is within the error, except for the upstream detector. At this position the detector specific energy cut-off and the shift of the particle spectrum to lower energies leads to a low statistics in the number of particles that contribute to the detector signal. The comparison of an experiment in a $400 \mathrm{GeV} / \mathrm{c}$ proton beam is in agreement with the simulation. A comparison of a gamma ray measurement (Cs137) with the simulated detector response was within $36 \%$. An experiment at the HERA proton beam dump to estimate the error of Geant 4 far transverse hadronic shower tail simulations was performed. The comparison of measurement and simulation close to the shower maximum shows a good agreement. At the other positions the simulation differs from the measurement. Over a period of 6 months the LHC BLM electronics was successfully used for this experiment. Further verification tests at the Svedberg Laboratory, Uppsala University (Sweden) [7] are planed for November 2006.

\section{ACKNOWLEDGMENT}

The authors would like to to acknowledge the contributions of various members of the LHC BLM section [9].

\section{REFERENCES}

[1] R. Schmidt et al., Protection of the CERN large hadron collider, New Journal of Physics, accepted for publication, 2006.

[2] E. B. Holzer et al., Beam Loss Monitoring System for the LHC, IEEE NSS-MIC '05, Puerto Rico, CERN-AB-2006-009 BI, 2005.

[3] Geant4 collaboration, Geant4 Simulation Toolkit, http://geant4. web. cern. ch/geant 4, viewed October 30, 2006.

[4] International Commission on Radiation Units and Measurements, Average Energy Required To Produce An Ion Pair, ICRU Report 31, Washington D.C., (1979)

[5] NIST (National Institute of Standard and Technology) Standard Reference Database 124, Stopping-Power and Range Tables for Electrons, Protons, and Helium Ions, http://physics.nist.gov/PhysRefData/ Star/Text/contents.html, viewed March 3, 2006.

[6] H. Vincke, N. Aguilar, D. Forkel-Wirth, M. Pangallo, D. Perin, M. Renou, C. Theis, Measurements and Simulations of the PMI Chamber Response to the Radiation Field inside the CERF Target Area, CERN EDMS No.: 457013, (CERN-SC-2004-025-RP-TN).

[7] A. V. Prokofiev, O. Bystrm, C. Ekstrm, V. Ziemann, J. Blomgren, S. Pomp, M. Oesterlund, and U. Tippawan, A New Neutron Beam Facility at $T S L$, International Workshop on Fast Neutron Detectors and Applications, University of Cape Town, South Africa, (FNDA2006 016), 2006.

[8] W. Friesenbichler, Development of the Readout Electronics for the Beam Loss Monitors of the LHC, CERN-THESIS-2002-028, Diplomarbeit, Fachhochschule Wr. Neustadt, 2002.

C. Zamantzas et al., The LHC beam loss monitoring system's real-time data analysis card, CERN-AB-2005-082, DIPAC 05, Lyon, France, 2005. C. Zamantzas, The Design, Construction, Test and Integration of the LHC Beam Loss Monitor Threshold Comparator (BLMTC), PhD Thesis, Brunel University, London, UK, 2006.

C. Zamantzas, B. Dehning, E. Effinger, J. Emery, G. Ferioli, An FPGA Based Implementation for Real-Time Processing of the LHC Beam Loss Monitoring System's Data, IEEE NSS-MIC '06, San Diego, IEEE Trans. Nucl. Sci., submitted for publication, 2006.

[9] www.cern.ch/blm 